\title{
Application of Exponential Kernel to Laplace Transform
}

\author{
Sami M. AL-Jaber \\ Department of Physics, An-Najah National University, Nablus, Palestine \\ Email: jaber@najah.edu
}

How to cite this paper: AL-Jaber, S.M. (2019) Application of Exponential Kernel to Laplace Transform. Journal of Applied Mathematics and Physics, 7, 1126-1130. https://doi.org/10.4236/jamp.2019.75075

Received: April 26, 2019

Accepted: May 24, 2019

Published: May 27, 2019

Copyright (C 2019 by author(s) and Scientific Research Publishing Inc. This work is licensed under the Creative Commons Attribution International License (CC BY 4.0).

http://creativecommons.org/licenses/by/4.0/

\begin{abstract}
In this paper, the exponential decreasing kernel is used in Laplace integral transform to transform a function from a certain domain to another domain. It is shown, in a rigorous way, that the Laplace transform of the delta function is exactly one half rather than one, as it is believed. In addition, when this kernel is used in integral transform of attractive and repulsive Coulomb potential, it yields a finite definite value at the point of singularity.
\end{abstract}

\section{Keywords}

Kernels, Integral Transforms, Laplace Transforms, Singularity

\section{Introduction}

Usually, kernels determine an implicit map that transforms a function or data from the input space to a feature space, and therefore determine its distribution in the latter space. This is usually accomplished through integral transforms. Some of the well-known kernels include the polynomial, exponential and Gaussian kernels. In particular, the exponential kernel through Laplace transform has been widely used over the years [1]-[6]. The Laplace transform is defined to transform a function from a space, say $x \in[0, \infty)$ to a space, say $s \in(0, \infty)$. Finding the Laplace transform of a function and its properties is normally discussed in standard mathematical physics books [7] [8]. An interesting function (more precisely a limit of some distribution) is the Dirac delta function, which has been in use in different settings [9]-[15]. The value of the Laplace transform of Delta-function can be found in mathematical physics books [8], where it is claimed that this value is one. We believe that the approach used to obtain this result is oversimplified and not rigorous. Therefore, one main object of this paper is to present a rigorous proof, through the use of a decreasing exponential 
kernel, and show that the correct value of Laplace transform of the delta function is exactly one half. The second part of this paper is to apply the decreasing exponential kernel to a discontinuous function. In particular, we consider a function with repulsive Coulomb-like form on the positive real axis, and with attractive Coulomb-like form on the negative real axis. This function is singular at the origin, and its right-hand and left-hand limits towards the origin are $+\infty$ and $-\infty$ respectively. It is shown, with this decreasing exponential kernel, that the value of this function is exactly zero which is the average between its limiting values at the origin. The last section of this paper is devoted for conclusion and discussion.

\section{The Laplace Transform of Delta-Function}

Consider the decreasing exponential kernel $\mathrm{e}^{-s|x|}$ and the delta function $\delta(x)$. Our aim is to derive the Laplace transform of $\delta(x)$ by applying this kernel to the integral;

$$
\int_{-\infty}^{\infty} \mathrm{e}^{-s|x|} \delta(x) \mathrm{d} x, \quad s>0
$$

Due to the well-known property of the delta-function, namely

$$
\int_{-\infty}^{\infty} f(x) \delta(x-a) \mathrm{d} x=f(a)
$$

Equation (1) becomes,

$$
\int_{-\infty}^{\infty} \mathrm{e}^{-s|x|} \delta(x) \mathrm{d} x=\mathrm{e}^{0}=1 .
$$

Splitting the integral into two parts, we get

$$
\int_{-\infty}^{\infty} \mathrm{e}^{-s|x|} \delta(x) \mathrm{d} x=\int_{-\infty}^{0} \mathrm{e}^{-s|x|} \delta(x) \mathrm{d} x+\int_{0}^{\infty} \mathrm{e}^{-s|x|} \delta(x) \mathrm{d} x .
$$

In the first integral on the left-hand side, $|x|=-x$, and by letting $x \rightarrow-x$, we get

$$
\int_{-\infty}^{0} \mathrm{e}^{-s|x|} \delta(x)=\int_{\infty}^{0} \mathrm{e}^{-s x} \delta(-x)(-\mathrm{d} x)=\int_{0}^{\infty} \mathrm{e}^{-s x} \delta(x) \mathrm{d} x .
$$

Note that, in the last step, we used the fact that $\delta(-x)=\delta(x)$, since it is even. So upon the substitution of Equation (5) into Equation (4), one gets

$$
\int_{-\infty}^{\infty} \mathrm{e}^{-s|x|} \delta(x) \mathrm{d} x=2 \int_{0}^{\infty} \mathrm{e}^{-s x} \delta(x) \mathrm{d} x .
$$

The Laplace transform of a function $f(x)$ is defined as

$$
\mathcal{L}\{f(x)\}=f(s)=\int_{0}^{\infty} \mathrm{e}^{-s x} f(x) \mathrm{d} x .
$$

Therefore, Equation (6) yields

$$
\int_{-\infty}^{\infty} \mathrm{e}^{-s|x|} \delta(x) \mathrm{d} x=2 \mathcal{L}\{\delta(x)\} .
$$

Hence, the use of Equation (3) gives the Laplace transform of $\delta(x)$, namely

$$
\mathcal{L}\{\delta(x)\}=\frac{1}{2} .
$$


The problem with the derivation of the unity value of the Laplace transform of the delta function, which is found in the literature [8], is overlooked at the lower limit $(x=0)$ in the definition of the Laplace transform. The point $x=0$ separates the positive and the negative parts of the $x$-axis. So, when applying Equation (2), one must ensure that the point $x=a$ must be totally included in the range of integration. This is not satisfied for the present case, and therefore one has to examine the whole domain of the delta function. This is the main essence of our derivation.

\section{Application of the Exponential Kernel to Coulomb-Like Function}

Discontinuous functions arise in some physical situations and usually one has to determine the value of this function at its point of discontinuity. Examples of these problems are the electric field at charged conducting sphere [16], the energy loss in the two capacitor problem [17] and Fermi-Dirac distribution [18]. Here, we consider a Coulomb-like potential (attractive and repulsive on negative and positive real axis respectively). This kind of function is discontinuous at the origin. We will show that this function converges to its average value at its singular point $(r=0)$.

In this section, we apply the decreasing exponential kernel to the Coulomb-like function which is given by

$$
f(r)=\left\{\begin{array}{ll}
\frac{1}{r} & r>0 \\
-\frac{1}{r} & r<0
\end{array} .\right.
$$

Consider the integral,

$$
\int_{-\infty}^{\infty} \mathrm{e}^{-s|r|} f(r) \mathrm{d} r=\int_{-\infty}^{0} \mathrm{e}^{s r}\left(-\frac{1}{r}\right) \mathrm{d} r+\int_{0}^{\infty} \mathrm{e}^{-s r}\left(\frac{1}{r}\right) \mathrm{d} r .
$$

Letting $r \rightarrow-r$ in the first integral of the left-hand side of the above equation, we get

$$
\begin{aligned}
\int_{-\infty}^{\infty} \mathrm{e}^{-s|r|} f(r) \mathrm{d} r & =-\int_{\infty}^{0} \mathrm{e}^{-s r} \frac{1}{r} \mathrm{~d} r+\int_{0}^{\infty} \mathrm{e}^{-s r} \frac{1}{r} \mathrm{~d} r \\
& =2 \int_{0}^{\infty} \mathrm{e}^{-s r} \frac{1}{r} \mathrm{~d} r=2 \mathcal{L}\left\{\frac{1}{r}\right\} .
\end{aligned} .
$$

Note that the function $f(r)$ is odd and the kernel is even so that the integral on the left-hand side of Equation (12) is zero. Two conclusions from the above equation are drawn: The first one is that the Laplace transform $\mathcal{L}(1 / r)=0$. For the second conclusion, we first observe that the limit of the integral on the left-hand side of Equation (12) as $s \rightarrow \infty$, the kernel $\mathrm{e}^{-s|r|} \rightarrow 0$ except at the point $r=0$, at which it is just a constant. In this case, to ensure the vanishing of the integral on the left-hand side of Equation (12), the function $f(r)$ must vanish at the origin, i.e. $f(0)=0$. It is noticed that $\lim _{r \rightarrow r^{+}} f(r)=\infty$ and $\lim _{r \rightarrow r^{-}} f(r)=-\infty$, so that the average between these two limiting values is zero. 
Therefore, our second conclusion is that the value of the function at its point of discontinuity converges to its average value between its two limiting values at that point.

\section{Conclusion and Discussion}

In this paper, a decreasing exponential kernel was used to derive the correct value of the Laplace transform of the delta function which is found to be one half. We also applied this type of kernel to a function which has a Coulomb-like form. Two conclusions of this application to such function were drawn: The first is that the Laplace transform of $\left(\frac{1}{r}\right)$ is zero and the second is that the value of this function at its point of discontinuity is the average value between its two limiting values about that point.

\section{Conflicts of Interest}

The author declares no conflicts of interest regarding the publication of this paper.

\section{References}

[1] Tsaur, J. and Wang, P. (2014) A Universal Laplace-Transform Approach to Solving Schrödinger Equation for All Solvable Models. European Journal of Physics, 35, Article ID: 015006. https://doi.org/10.1088/0143-0807/35/1/015006

[2] Pimental, D.R.M. and de Castro, A.S. (2013) A Laplace Transform Approach to the Quantum Harmonic Oscillator. European Journal of Physics, 34, 199. https://doi.org/10.1088/0143-0807/34/1/199

[3] Penson, K.A. and Gorska, K. (2016) On the Properties of Laplace Transform Originating from One-Sided From Levy Stable Laws. Journal of Physics A: Mathematical and Theoretical, 49, Article ID: 065201. https://doi.org/10.1088/1751-8113/49/6/065201

[4] Kryzhniy, V.V. (2006) Numerical Inversion of Laplace Transform: Analysis via Regularized Analytic Continuation. Inverse Problems, 22, 579-597. https://doi.org/10.1088/0266-5611/22/2/012

[5] Viaggiu, V.V. (2017) Axial and Polar Gravitational Wave Equations in a de Sitter Expanding Universe by Laplace Transform. Classical and Quantum Gravity, 34, Article ID: 035018. https://doi.org/10.1088/1361-6382/aa5570

[6] Gonzalez, F., Saiz, J.M., Moreno, F. and Valle, R.J. (1992) Application of Laplace Transform Method to Binary Mixtures of Spherical Particles in Solution For Low Scattering Intensity. Journal of Physics D: Applied Physics, 25, 357-361. https://doi.org/10.1088/0022-3727/25/3/003

[7] Boas, M.L. (2005) Mathematical Methods in the Physical Sciences. Wiley \& Sons, Hoboken.

[8] Arfken, G.B. and Weber, H.J. (2005) Mathematical Physics for Physicists. Elsevier Academic Press, Amsterdam.

[9] Chua, C.-K., Liu, T.-U. and Wong, G.-G. (2018) Time-Independent Green's Function of a Quantum Simple Harmonic Oscillator System and Solutions with Additional Generic Delta-Function Potentials. Journal of Physics Communications, 2, 
Article ID: 035007. https://doi.org/10.1088/2399-6528/aa9eeb

[10] Galapon, E.A. (2009) Delta-Convergent Sequences That Vanish at the Support of the Limit Delta Function. Journal of Physics A: Mathematical and Theoretical, 42, Article ID: 175201. https://doi.org/10.1088/1751-8113/42/17/175201

[11] Parker, E. (2017) An Apparent Paradox Concerning the Field of a Dipole. European Journal of Physics, 38, Article ID: 025205. https://doi.org/10.1088/1361-6404/aa55a6

[12] Demirlab, E. (2005) Bound States of n-Dimensional Harmonic Oscillator Decorated by Delta-Functions. Journal of Physics A: Mathematical and Theoretical, 38, 4783-4793. https://doi.org/10.1088/0305-4470/38/22/003

[13] Zhong, M.A. and Yang, C.N. (2010) Bosons or Fermions in 1D Power Potential Trap with Repulsive Delta Function Interactions. Chinese Physics Letters, 27, Article ID: 090505. https://doi.org/10.1088/0256-307X/27/9/090505

[14] Dmitriev, D.V. and Krivnov, V. (2018) Heisenberg-Ising Delta-Chain with Bond Alternation. Journal of Physics. Condefnsed Matter, 30, Article ID: 385803. https://doi.org/10.1088/1361-648X/aadb72

[15] Tracy, C.A. and Widom, H. (2016) On the Ground State Energy on the $\delta$-Function Bose Gas. Journal of Physics A: Mathematical and Theoretical, 49, Article ID: 294001. https://doi.org/10.1088/1751-8113/49/29/294001

[16] Griffiths, D.J. (1999) Introduction to Electrodynamics. Cambridge University Press, Cambridge.

[17] AL-Jaber, S.M. and Salih, S. (2000) Energy Considerations in the Two-Capacitor Problem. European Journal of Physics, 41, 341. https://doi.org/10.1088/0143-0807/21/4/307

[18] Pathria, R.K. and Peale, P.D. (2011) Statistical Mechanics. Academic Press, New York. 